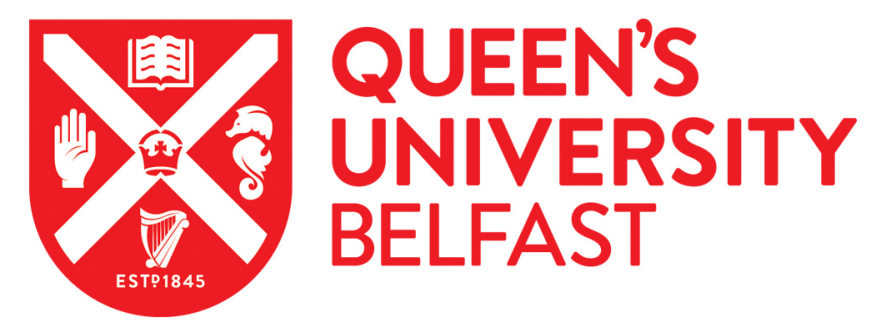

\title{
Probing spin-orbit-interaction-induced electron dynamics in the carbon atom by multiphoton ionization
}

Rey, H. F., \& Van Der Hart, H. W. (2014). Probing spin-orbit-interaction-induced electron dynamics in the carbon atom by multiphoton ionization. Physical Review A (Atomic, Molecular, and Optical Physics), 90, [033402]. https://doi.org/10.1103/PhysRevA.90.033402

Published in:

Physical Review A (Atomic, Molecular, and Optical Physics)

Document Version:

Publisher's PDF, also known as Version of record

Queen's University Belfast - Research Portal:

Link to publication record in Queen's University Belfast Research Portal

Publisher rights

@2014 American Physical Society

\section{General rights}

Copyright for the publications made accessible via the Queen's University Belfast Research Portal is retained by the author(s) and / or other copyright owners and it is a condition of accessing these publications that users recognise and abide by the legal requirements associated with these rights.

Take down policy

The Research Portal is Queen's institutional repository that provides access to Queen's research output. Every effort has been made to ensure that content in the Research Portal does not infringe any person's rights, or applicable UK laws. If you discover content in the Research Portal that you believe breaches copyright or violates any law, please contact openaccess@qub.ac.uk. 


\title{
Probing spin-orbit-interaction-induced electron dynamics in the carbon atom by multiphoton ionization
}

\author{
H. F. Rey ${ }^{*}$ and H. W. van der Hart \\ Centre for Theoretical Atomic, Molecular and Optical Physics, School of Mathematics and Physics, Queen's University Belfast, \\ Belfast BT7 1NN, United Kingdom \\ (Received 16 May 2014; published 3 September 2014)
}

\begin{abstract}
We use $R$-matrix theory with time dependence (RMT) to investigate multiphoton ionization of ground-state atomic carbon with initial orbital magnetic quantum number $M_{L}=0$ and $M_{L}=1$ at a laser wavelength of $390 \mathrm{~nm}$ and peak intensity of $10^{14} \mathrm{~W} / \mathrm{cm}^{2}$. Significant differences in ionization yield and ejected-electron momentum distribution are observed between the two values for $M_{L}$. We use our theoretical results to model how the spin-orbit interaction affects electron emission along the laser polarization axis. Under the assumption that an initial $\mathrm{C}$ atom is prepared at zero time delay with $M_{L}=0$, the dynamics with respect to time delay of an ionizing probe pulse modeled by using RMT theory is found to be in good agreement with available experimental data.
\end{abstract}

DOI: 10.1103/PhysRevA.90.033402

PACS number(s): $32.80 . \mathrm{Rm}, 31.15 . \mathrm{A}-$

\section{INTRODUCTION}

Over the last 15 years great advances have been made in the development and application of experimental laser techniques on the subfemtosecond timescale $\left(1 \mathrm{fs}=10^{-15} \mathrm{~s}\right)$ [1]. These advances open the prospect of the detailed study and potential guiding of electron motion in atoms and molecules. Several methods have been applied to monitor the electron dynamics, such as pump-probe spectroscopy using few-cycle pulses, through which, for example, the angular correlation between sequential ionization steps was investigated [2]. Another example is transient absorption spectroscopy, in which, for example, sequential ionization dynamics of $\mathrm{Kr}$ was investigated [3]. This experiment demonstrated the influence of the spin-orbit interaction on the $\mathrm{Kr}^{+}$dynamics when sufficiently short ionization pulses are employed.

Electron motion in a general atom can be regarded as the complement of atomic structure via the time-energy uncertainty principle. In the atomic structure of light atoms, relativistic interactions, such as spin-orbit coupling, are normally assumed to be negligible. However, in heavier atoms, this assumption no longer holds. As a consequence, it can be assumed that spin-orbit dynamics may have a relatively minor effect on electron dynamics of light atoms on the timescale of a short laser pulse, whereas this dynamics will become important when heavier atoms are considered. That does not mean, however, that spin-orbit dynamics is irrelevant for light atoms: when light atoms interact with a sequence of two light pulses with a long time delay between them, spin-orbit dynamics can significantly affect the intermediate state [4,5].

Changes in the atomic state induced by spin-orbit interaction can significantly affect the subsequent atomic dynamics [6]. Total angular momentum $J=L+S$, with $L$ being the total orbital angular momentum and $S$ being the total spin, is conserved, as is its projection on the $z$ axis, $M_{J}=M_{L}+M_{S}$. However, although $M_{J}$ is conserved, its components $M_{L}$ and $M_{S}$ are not. Hence, the spin-orbit interaction can change, for example, a $J M_{J} L S$ state with $M_{L}=0$ and $M_{S}=+1 / 2$ into a $J M_{J} L S$ state with $M_{L}=+1$ and $M_{S}=-1 / 2$. This change

\footnotetext{
*h.rey@qub.ac.uk
}

in orbital magnetic quantum number can have a noticeable effect on the atomic dynamics. For example, in harmonic generation of noble-gas ions $\mathrm{Ne}^{+}$and $\mathrm{Ar}^{+}$, the harmonic yields for $M_{L}=0$ were reduced by a factor of two to four compared to $M_{L}= \pm 1[7,8]$.

Recently, pump-probe experiments have been carried out to investigate the spin-orbit-induced dynamics in the $\mathrm{C}$ ground state following photodetachment of $\mathrm{C}^{-}[4,5]$. In these studies, an initial $\mathrm{C}$ atom was formed by photodetachment of $\mathrm{C}^{-}$. This residual $\mathrm{C}$ atom is left in a superposition of ground state ${ }^{3} P^{e}$ levels with different $J$. The energy splitting between the different $J$ levels then leads to dynamics within the different $M_{L}$ levels, which can be observed through differences in the measured ejected-electron momentum spectra between parallel polarization of the pump and probe pulses and perpendicular polarization of the pulses. These differences were shown to vary with a period of 760 to $2000 \mathrm{fs}$, as can be deduced from the energy difference between the $J$ levels of the $\mathrm{C}^{3} P^{e}$ ground state, $2.0 \mathrm{meV}$ between the lower $J=0$ and the $J=1$ level, and $3.4 \mathrm{meV}$ between the $J=1$ and $J=2$ level [9].

From a theoretical perspective, the influence of relativistic interactions on ultrafast atomic dynamics has already been the subject of theoretical investigation [10,11]. However, it would be very useful to develop this capability in other theoretical methods and techniques as well, such as, for example, time-dependent $R$-matrix theory [12-15]. The most recent implementation of time-dependent $R$-matrix theory, named $R$-matrix theory including time dependence (RMT), is the most efficient implementation for large-scale studies because it provides better stability when many angular momenta are included and provides better scope for exploitation of massively parallel computing facilities. The first step in this development is to verify that initial states with $M_{L} \neq 0$ can be investigated accurately by using the RMT approach. However, whereas the previous implementation of time-dependent $R$ matrix theory has already been applied to the investigation of dynamics for atoms with $M_{L} \neq 0[7,8,16]$, up to now RMT theory has only been applied to systems with $M_{L}=0[17,18]$.

In this report, we demonstrate that RMT theory can be applied to the study of atoms with an initial $M_{L} \neq 0$. We apply the theory to investigate ejected-electron momentum 
distributions for the multiphoton ionization of $\mathrm{C}$ atoms in short $390 \mathrm{~nm}$ laser pulses with a peak intensity of $10^{14} \mathrm{~W} / \mathrm{cm}^{2}$ for both $M_{L}=0$ and $M_{L}=1$. We will explain the significant changes in these momentum distributions between the two values of $M_{L}$. We then use these momentum distributions to model the observation of spin-orbit dynamics in $\mathrm{C}$ as a function of time delay $\tau$ for the ionization pulse, under the assumption that a ground-state $\mathrm{C}$ atom is created at time $t=0$ by emission of an $m_{\ell}=0$ electron from ground-state $\mathrm{C}^{-}$. Since the spin-orbit dynamics in $\mathrm{C}$ occurs on timescales longer than the duration of the $390 \mathrm{~nm}$ laser pulse, which takes $1.3 \mathrm{fs}$ per cycle, it can be assumed that no spin-orbit dynamics occurs during the interaction with the laser pulse. The time-delayed initial state of $\mathrm{C}$ can then be regarded as a superposition of states with $M_{L}=1, M_{L}=0$, and $M_{L}=-1$.

The organization of the report is as follows: In Sec. II, we give a brief overview of RMT theory, including a description of changes required to treat nonzero $M_{L}$. The specific parameters used in the present calculations, including a description of the $\mathrm{C}^{+}$basis set, are also included. In Sec. III, we present our calculated ejected-electron momentum distributions along with selected photoelectron energy spectra focusing on the differences seen for $M_{L}=0$ and $M_{L}=1$. We then model the differences in the ejected-electron momentum spectra that would be induced by spin-orbit dynamics and compare these with the experimental findings [4,5]. Finally in Sec IV, we report our conclusions.

\section{METHODS}

\section{A. The RMT approach}

In this report, we employ the RMT approach to investigate multiphoton ionization of neutral carbon and model the ejected electron dynamics. The RMT method builds upon traditional $R$-matrix theory $[19,20]$ by separating the description of the system into two distinct regions: an inner region and an outer region. In the inner region, the wave function is expressed in terms of a multi-electron $R$-matrix basis description within a confined spatial region surrounding the nucleus [20]. In the outer region, the full multi-electron wave function is described as a direct product of a channel function and a finite-difference representation of the single-electron radial wave function for an outer electron found only outside the confined spatial region $[14,15]$. The channel function comprises the wave function of a residual-ion state coupled with the spin and angular-momentum functions of the ejected electron.

A key feature of the RMT approach is the connection between the inner and outer regions. To provide information about the inner-region wave function to the outer region, the outer-region grid is extended into the inner region. The inner-region radial wave function is then evaluated on this grid extension and made available to the outer region. In order to provide outer-region wave-function information to the inner region, time derivatives of the outer-region wave function are evaluated at the inner-region boundary and are then provided to the inner region. Full details about the link between the two regions are provided in Refs. [14,15].

Time propagation within the RMT approach is carried out through Arnoldi propagators [21]. Through the grid extension into the inner region, all the necessary information for propagation of the outer-region wave function is available on the outer-region grid, and the propagation through an Arnoldi propagator is relatively straightforward. In the inner region, however, different Arnoldi propagators are needed for the wave function and for each outer-region time derivative at the boundary. All time derivatives are therefore propagated separately, and the full time-propagated wave function is obtained by combining all the separate propagation terms $[14,15]$.

The implementation of the RMT codes to date was limited to systems with an initial angular momentum $L=0$ and, consequently, $M_{L}=0$. However, the ground state of $\mathrm{C}$ has ${ }^{3} P^{e}$ symmetry, which can have a total magnetic quantum number $M_{L}=-1,0$, or 1 . The value of $M_{L}$ significantly affects the calculations. According to dipole selection rules, only transitions with $\Delta L= \pm 1$ are allowed for $M_{L}=0$. On the other hand, for $M_{L}=1$, transitions with $\Delta L=0$ are allowed in addition to $\Delta L= \pm 1$. As a consequence, for $M_{L}=0$, only a single parity needs to be included for each value of $L$, whereas for $M_{L}=1$, both parities need to be included for each value of $L$. More symmetries are therefore available for $M_{L}=1$ and the calculations approximately double in size. In addition, we need to take into account that all symmetries can now potentially interact with three other symmetries, rather than two. Within the parallel implementation of the RMT codes, this requires additional communication between message-passinginterface tasks. However, since the propagation involves only matrix-vector multiplications, no reordering of the symmetry blocks is required, in contrast to the previous implementation of time-dependent $R$-matrix theory [16].

\section{B. The description of $\mathbf{C}$}

To study the influence of the initial value of $M_{L}$ values on the multiphoton ionization dynamics of $\mathrm{C}$, we employ an $R$-matrix basis set previously employed to investigate photoionization of $\mathrm{C}$ [22]. In this basis set, all possible residual-ion states are considered that can be formed using $2 s$ and $2 p$ orbitals:

$$
\begin{aligned}
n \hbar \omega+\mathrm{C}\left(2 s^{2} 2 p^{2}{ }^{3} P^{e}\right) & \rightarrow \mathrm{C}^{+}\left(2 s^{2} 2 p^{2} P^{o}\right)+e^{-} \\
& \rightarrow \mathrm{C}^{+}\left(2 s 2 p^{2} 2,4 P^{e},{ }^{2} D^{e},{ }^{2} S^{e}\right)+e^{-} \\
& \rightarrow \mathrm{C}^{+}\left(2 p^{3}{ }^{4} S^{o},{ }^{2} D^{o},{ }^{2} P^{o}\right)+e^{-}
\end{aligned}
$$

The $1 s, 2 s$, and $2 p$ orbitals building these three ionic configurations are given by the $1 s, 2 s$, and $2 p$ Hartree-Fock orbitals for the ${ }^{2} P^{o}$ ground state of the ion [23]. To improve the description of the $\mathrm{C}^{+}$eigenstates, the basis set also includes $\overline{3 s}, \overline{3 p}$, and $\overline{3 d}$ pseudo-orbitals orthogonal to the Hartree-Fock orbitals. The $\mathrm{C}^{+}$basis set is then expanded by allowing single excitations from the $2 s^{2} 2 p, 2 s 2 p^{2}$, and $2 p^{3}$ configurations to these pseudo-orbitals. Energies of the lowest-five eigenstates of $\mathrm{C}^{+}$are given in Table $\mathrm{I}$ and compared with literature values [9]. The energies of the $2 p^{3}$ states are not given because they lie above the $2 s^{2} 3 s$ states, which have been excluded from the present calculations. The energies agree exactly with those obtained in Ref. [22], apart from the energy for the $2 s 2 p^{2}{ }^{2} S^{e}$ state. This is most likely due to a small difference in the configuration-interaction (CI) expansion used 
TABLE I. $\mathrm{C}^{+}$threshold energies relative to the ground state of C, compared to literature values [9]. Energies are not given for the $2 p^{3}$ states as these lie in between the $2 s^{2} 3 \ell$ and $2 s^{2} 4 \ell$ states. Apart from the energy for the $2 s 2 p^{2} S^{e}$ state, all present energies agree with those in Ref. [22].

\begin{tabular}{lcc}
\hline \hline $\mathrm{C}^{+}$state & $\begin{array}{c}\text { Present energy } \\
(\mathrm{eV})\end{array}$ & $\begin{array}{c}\text { Literature [9] } \\
(\mathrm{eV})\end{array}$ \\
\hline $2 s^{2} 2 p^{2} P^{o}$ & 11.366 & 11.262 \\
$2 s 2 p^{2}{ }^{4} P^{e}$ & 16.430 & 16.592 \\
$2 s 2 p^{2}{ }^{2} D^{e}$ & 20.962 & 20.547 \\
$2 s 2 p^{2} S^{e}$ & 24.196 & 23.220 \\
$2 s 2 p^{2}{ }^{2} P^{e}$ & 25.706 & 24.976 \\
\hline \hline
\end{tabular}

for this state, which includes six basis functions in the present calculations, whereas the one reported in Ref. [22] includes five basis functions. The table demonstrates that the lowest-two thresholds are within $0.2 \mathrm{eV}$ of the literature values, whereas the differences increase rapidly for the higher states. This is not surprising because the $2 s 2 p^{2}{ }^{2} D^{e}$ and ${ }^{2} S^{e}$ states will interact strongly with the physical $2 s^{2} 3 d$ and $2 s^{2} 3 s$ states, respectively. The $\overline{3 s}$ and $\overline{3 d}$ orbitals used in the present calculations are pseudo orbitals and will therefore not accurately reflect the $3 \mathrm{~s}$ and $3 d$ orbitals in the physical states. Hence the interaction with $2 s^{2} 3 d$ and $2 s^{2} 3 s$ will not be described accurately, and the obtained threshold energies will be less accurate. Nevertheless, the most important threshold states in the present multiphoton calculations are the lowest two thresholds, and these are described with sufficient accuracy for the present purposes.

The $\mathrm{C}$ basis set is formed by combining the eigenstates for $\mathrm{C}^{+}$obtained given the basis set above with a basis set describing the continuum electron. The functions in this basis set are described in terms of $65 B$ splines of order $k=11$. This basis set is augmented by correlation functions, which are created by adding one of the input orbitals to the configurations used in the description of $\mathrm{C}^{+}$. It is further ensured that every function in the continuum basis set is orthogonal to any input orbital. The radius of the inner region was chosen to be $27 a_{0}$. This is larger than considered in the previous $\mathrm{C}$ photoionization calculations, because, at present, the initial state needs to be fully contained within the inner region in the RMT approach. The $\mathrm{C}$ ground-state energy has not been shifted.

In the multiphoton-ionization calculations, we investigate $\mathrm{C}$ atoms irradiated by a laser field with a wavelength of $390 \mathrm{~nm}$. The total pulse length is eight cycles, consisting of a threecycle $\sin ^{2}$ ramp-on, two cycles at peak intensity, followed by a three-cycle $\sin ^{2}$ ramp-off of the electric field. Following the end of the pulse, the wave function is propagated field free for another twelve cycles. For the present investigations, the peak intensity was chosen to be $10^{14} \mathrm{~W} / \mathrm{cm}^{2}$.

In the analysis of the final wave function, we only consider the outer-region wave function, because continuum electrons are assumed to have escaped the inner region after the propagation at the end of the pulse. Within the RMT approach, the outer-region wave function is described in terms of a residual ionic state plus spin and angular momentum of the outer electron coupled with a radial wave function for the outer electron on a discretized finite-difference grid. The finite-difference grid has a grid spacing of $\Delta r=0.15 a_{0}$ and extends to an outer radius of over $3900 a_{0}$.

As indicated earlier, time propagation within the RMT approach is achieved through the use of Arnoldi propagators [21]. In the present application, we use Arnoldi propagators of order 12 with a time step of $\Delta t=0.01$ atomic units.

\section{RESULTS}

The main goal in the present calculations is to study how spin-orbit dynamics affects the multiphoton-ionization characteristics of ground-state carbon atoms. Since the spinorbit dynamics in ground-state $\mathrm{C}$ is slow compared to the present photoionization dynamics, we can decouple the two types of dynamics and instead investigate how changes in the orbital magnetic quantum number $M_{L}$ are reflected in the multiphoton ionization characteristics. We can subsequently investigate how spin-orbit dynamics changes the magnetic sublevel populations in time, and then we derive the changes in the ejected-electron momentum spectra driven by the spin-orbit interaction.

A second objective of the present calculations is to investigate the convergence of the final-state populations in the different partial waves with the number of angular momenta retained in the calculation. We adopted as our convergence criterion that the final population in each outer channel should converge to within $0.01 \%$ of the population in the most populated channel. This convergence was achieved for a maximum angular momentum to be retained in the calculation, $L_{\max }$, of 53 . We also carried out calculation with different propagation orders and found no significant difference between propagation orders of 12 and 14. All calculations were therefore carried out by using an Arnoldi propagator of order 12 .

The main outcomes of the calculations are shown in Figs. 1 and 2, which show the ejected-electron momentum distributions obtained for ground-state C atoms irradiated by $390 \mathrm{~nm}$

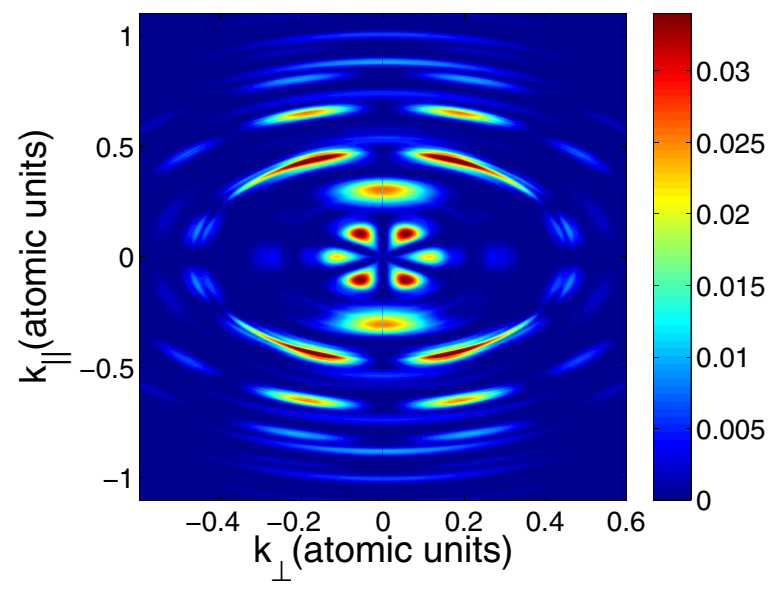

FIG. 1. (Color online) Ejected-electron momentum distribution in the $k_{x} k_{z}$ plane for $\mathrm{C}$ initially in the ground state with $M_{L}=0$, irradiated by an eight-cycle UV laser pulse with wavelength $390 \mathrm{~nm}$ and intensity $10^{14} \mathrm{~W} / \mathrm{cm}^{2}$. The distribution includes the emission of electrons towards the lowest two states of $\mathrm{C}^{+}$, which contribute over $99 \%$ to the total outer-region population in the present calculations. 


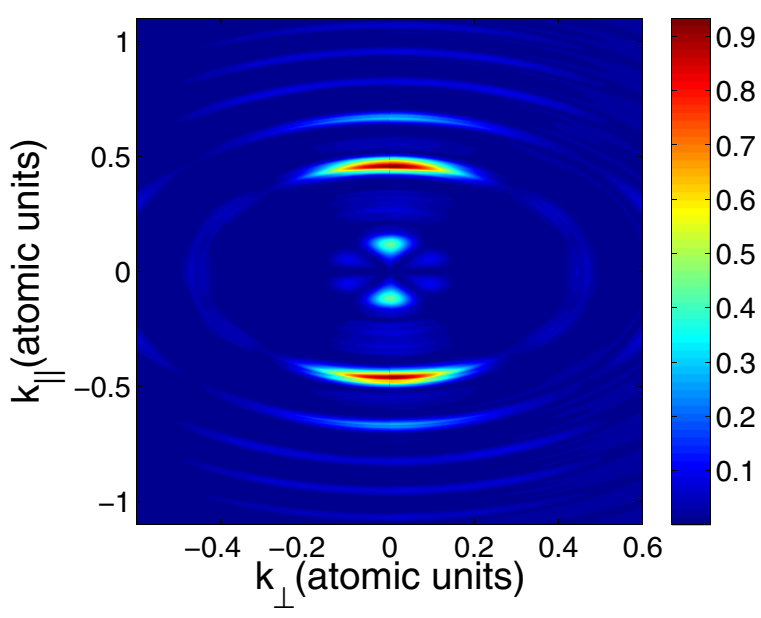

FIG. 2. (Color online) Ejected-electron momentum distribution in the $k_{x} k_{z}$ plane for $\mathrm{C}$ initially in the ground state with $M_{L}=1$, irradiated by an eight-cycle UV laser pulse with wavelength $390 \mathrm{~nm}$ and intensity $10^{14} \mathrm{~W} / \mathrm{cm}^{2}$. The distribution includes the emission of electrons towards the lowest two states of $\mathrm{C}^{+}$, which contribute over $99 \%$ to the total outer-region population in the present calculations.

light at an intensity of $10^{14} \mathrm{~W} / \mathrm{cm}^{2}$ for $M_{L}=0$ and $M_{L}=1$, respectively. The determination of the ejected-electron momentum distributions is, however, not straightforward at this intensity. At an aprroximate intensity of $0.95 \times 10^{13} \mathrm{~W} / \mathrm{cm}^{2}$, channel closing occurs, so that five photons are needed for ionization instead of four. However, if the bandwidth of the laser pulse is taken into account as well, both four-photon excitation of Rydberg states and four-photon ionization will occur simultaneously. To improve the separation of Rydbergstate population from continuum-state population, the system is propagated for twelve $390 \mathrm{~nm}$ cycles after the pulse has ended.

In the experimental analysis of the ejected-electron momentum distributions, however, the low-energy part of the distributions was not taken into account [4,5]. We have therefore chosen to do the same in the present analysis. At the end of the calculations, we obtain the outer-region part of the final-state wave function. The outer electron is decoupled from the residual ion, which enables us to extract a wave function for the outer electron associated with each residual-ion state. We then transform the wave function for the outer electron for distances larger than $108 a_{0}$ into the momentum representation under the assumption that the Coulomb potential can be neglected. This distance was chosen by examination of the final-state wave function. For distances greater than $108 a_{0}$, the final-state wave function has clear continuum-wave characteristics, whereas this is not the case for distances smaller than $108 a_{0}$. To examine the influence of our choice of distance, we carried out our analysis for other distances as well. We observe that, if $\mathrm{C}^{+}$is left in the ground state, this leads to quantitative convergence of the momentum spectra for momenta greater than about 0.30 atomic units, with a good qualitative description for lower momenta. The momentum of an electron absorbing five photons is about 0.48 atomic units, and this part of the spectrum of primary interest is not affected by our choice of distance. If $\mathrm{C}^{+}$is left in the lowest excited state, the procedure leads to a good quantitative description over the entire momentum spectrum due to the absence of threshold photoelectrons. The momentum of an electron absorbing the minimum number (six) of photons is about 0.30 atomic units.

Since the $\mathrm{C}$ ground state has ${ }^{3} P^{e}$ symmetry, Figs. 1 and 2 show the momentum distributions for an initial orbital magnetic quantum number $M_{L}=0$ and $M_{L}=1$, respectively. The figures clearly show great differences between the two momentum distributions associated with the change of magnetic quantum number. For $M_{L}=1$, almost all the ionization is aligned along the laser polarization axis, whereas for $M_{L}=0$, two different contributions can be seen, one aligned along the laser polarization axis and one at an angle to it.

Two different distributions can be seen in the ejectedelectron momentum distributions for an initial $M_{L}=0$ in Fig. 1. These are associated with different residual-ion states of $\mathrm{C}^{+}$or, alternatively, the ejection of different electrons. Emission along the laser polarization axis involves emission of a $2 s$ electron, leaving $\mathrm{C}^{+}$in the excited $2 s 2 p^{24} P^{e}$ state, whereas emission away from the laser polarization axis involves emission of a $2 p$ electron leaving $\mathrm{C}^{+}$in its $2 s^{2} 2 p^{2} P^{o}$ ground state. For $M_{L}=0$, the total population beyond a distance of $108 a_{0}$, used in the determination of Fig. 1 , in $2 p$ emission channels, is $2.86 \%$, whereas the one in channels leaving $\mathrm{C}^{+}$in the $2 s 2 p^{2}{ }^{4} P^{e}$ state is $0.55 \%$. The total population in the outer region at the end of the calculation is $4.73 \%$, with $4.16 \%$ associated with the $\mathrm{C}^{+}$ground state and $0.56 \%$ with the first-excited state of $\mathrm{C}^{+}$. Higher-lying states of $\mathrm{C}^{+}$account for less than $0.4 \%$ of the total population in the outer region, and therefore the contribution to the momentum distributions from outgoing electrons attached to these states is assumed to be negligible. These populations indicate that, although the majority of the population in the outer region is assigned to ionization, a significant amount of population may be associated with Rydberg-state population. The main reason for this is the proximity of the intensity used in the calculations to a channel-closing intensity.

The same two distributions also make up the ejectedelectron momentum distributions for an initial magnetic quantum number $M_{L}=1$, shown in Fig. 2. However, in this case, the momentum distribution is dominated by the emission of a $2 p$ electron along the laser polarization axis. Emission of a $2 s$ electron towards the $2 s 2 p^{2}$ state of $\mathrm{C}^{+}$is still possible, but it is not apparent in the present ejected-electron momentum distribution. For $M_{L}=1$, the total population in $2 p$ emission channels beyond a distance of $108 a_{0}$ is $19.56 \%$, whereas it is $1.06 \%$ for $2 s$ emission channels leaving $\mathrm{C}^{+}$in the $2 s 2 p^{24} P^{e}$ state. The total population in the outer region at the end of the calculation is $28.31 \%$, with $27.17 \%$ associated with the $\mathrm{C}^{+}$ground state and $1.07 \%$ associated with the first-excited state of $\mathrm{C}^{+}$. Higher-lying states of $\mathrm{C}^{+}$account for less than $0.25 \%$ of the population in the outer region, and therefore the contribution to the momentum distributions from outer-region channels associated with these states is again assumed to be negligible. Again, the total population in the outer region is noticeably larger than the population associated with ionization. It can be seen that the total population in the outer region has increased by more than a factor of six from the population obtained in the $M_{L}=0$ calculation, with an 
increase in the emission probability for a $2 p$ electron by a factor seven. The emission probability for a $2 s$ electron has also increased, but only by a factor of two.

The reason for the difference in the ejected-electron momentum distribution is that emission of $m_{\ell}=0$ electrons is strongly preferred in a linearly polarized laser field. For an initial magnetic quantum number $M_{L}=0$, symmetry prevents the emission of an electron with $m_{\ell}=0$, leaving $\mathrm{C}^{+}$in a $2 s^{2} 2 p^{2} P^{o}$ state with $M_{L^{\prime}}^{\prime}=M_{L}-m_{\ell}=0$. Since transitions with $\Delta L=0$ are not allowed for $M_{L}=0$, the only $2 s^{2} 2 p$ ionization channels available are of the form $2 s^{2} 2 p\left({ }^{2} P^{o}\right) \varepsilon \ell^{3} L$ with $\ell=L$. However, the Clebsch-Gordan coefficient $\mathrm{CG}(1 L L ; 000)$ is identical to 0 . On the other hand, for $M_{L}=1$, emission of a $2 p$ electron with $m_{\ell}=0$ is allowed, and this process dominates the ionization.

The contribution of emission of a $2 s$ electron to the total ionization demonstrates that above-threshold ionization is important for $\mathrm{C}$ at this combination of wavelength and intensity. It takes absorption of one extra photon to eject the $2 s$ electron. This importance of above-threshold ionization is also visible in the ejected-electron momentum distributions. For emission of the $2 p$ electron, three additional peaks at higher momentum can be seen for $M_{L}=0$ and one for $M_{L}=1$. For emission of a $2 s$ electron, however, another four peaks can be seen along the laser polarization axis for $M_{L}=0$.

To visualize the importance of above-threshold ionization in more detail, we can also investigate the photoelectron spectrum for a particular emission angle. The photoelectron energy spectrum associated with the $2 s^{2} 2 p^{2} P^{o}$ ground and ${ }^{4} P^{e}$ excited state of the residual $\mathrm{C}^{+}$ion are shown in Figs. 3 and 4 , respectively, for both $M_{L}=0$ and $M_{L}=1$. We have chosen an angle of $27^{\circ}$ with respect to the laser polarization axis for these energy spectra, because this allows these spectra to be studied with a reasonable magnitude for all four cases.

Figure 3 shows the photoelectron spectrum associated with the residual $\mathrm{C}^{+}$ion left in its ground state for initial $\mathrm{C}$ atoms in either $M_{L}=0$ or $M_{L}=1$. It can be seen that the overall magnitude of the photoelectron spectrum is significantly larger
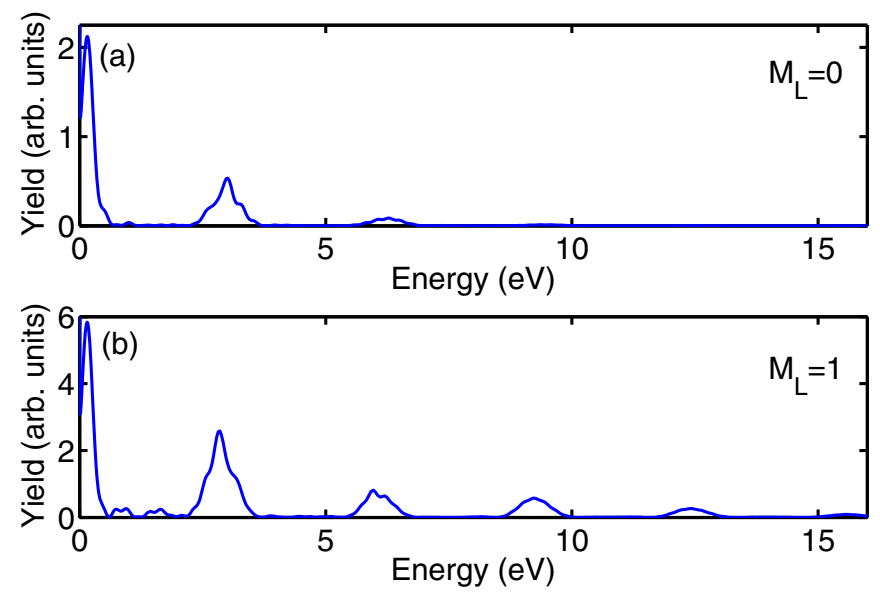

FIG. 3. (Color online) Photo-electron spectra for electrons leaving the residual $\mathrm{C}^{+}$ion in the ground state observed at an angle of $27^{\circ}$ with respect to the laser polarization axis for (a) $M_{L}=0$ and (b) $M_{L}=1$ in a laser field with a wavelength of $390 \mathrm{~nm}$ and peak intensity of $10^{14} \mathrm{~W} / \mathrm{cm}^{2}$.
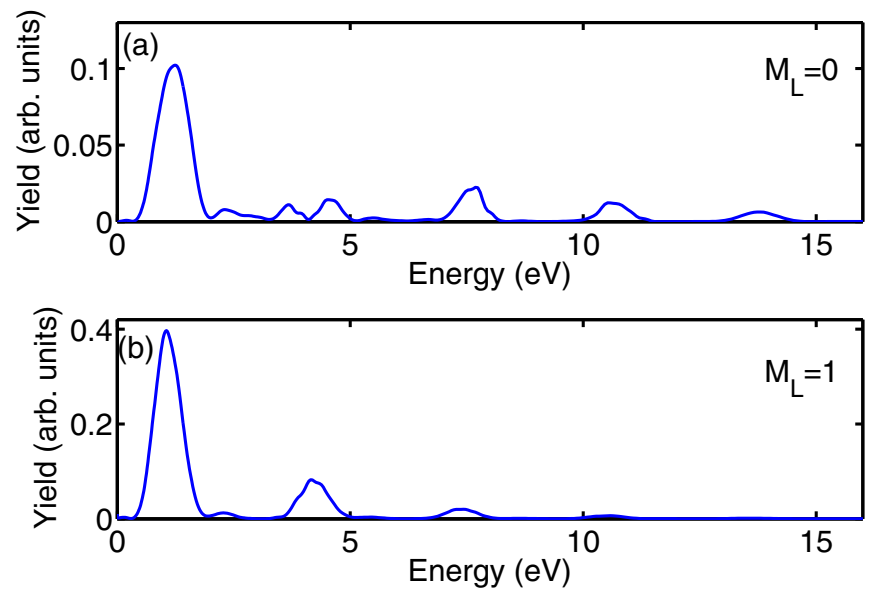

FIG. 4. (Color online) Photoelectron spectra for electrons leaving the residual $\mathrm{C}^{+}$ion in the excited $2 s 2 p^{2}{ }^{4} P^{e}$ state observed at an angle of $27^{\circ}$ with respect to the laser polarization axis for (a) $M_{L}=0$ and (b) $M_{L}=1$ in a laser field with a wavelength of $390 \mathrm{~nm}$ and peak intensity of $10^{14} \mathrm{~W} / \mathrm{cm}^{2}$.

for $M_{L}=1$ than it is for $M_{L}=0$, which is primarily due to the increase in ionization yield for $M_{L}=1$ : the peak at $3 \mathrm{eV}$ has increased by about a factor five. It can furthermore be seen that the number of above-threshold peaks is substantially larger for $M_{L}=1$ than for $M_{L}=0$. For $M_{L}=0$, the abovethreshold-ionization peaks drop off rapidly with increasing energy. For $M_{L}=1$, however, after a sharp drop for the second peak, the drop off for higher peaks is significantly slower. The origin probably lies with the dominant $m_{\ell}$ value of the ejected electron. For $M_{L}=0$, this is $m_{\ell}= \pm 1$, but it is $m_{\ell}=$ 0 for $M_{L}=1$. Since electrons with $m_{\ell}=0$ respond more strongly to the laser field than do electrons with $m_{\ell}= \pm 1$, it is not unexpected to see additional absorption peaks in the $2 p$ emission spectrum for $M_{L}=1$.

Figure 4 shows the photoelectron spectrum associated with the residual $\mathrm{C}^{+}$ion left in its lowest excited state for initial $\mathrm{C}$ atoms in either $M_{L}=0$ or $M_{L}=1$. This figure demonstrates that the probability of emission of a $2 s$ electron has increased for $M_{L}=1$ compared to $M_{L}=0$, even though the $2 s$ emission is not visible for $M_{L}=1$ in Fig. 2. The structure of the ejected-electron energy spectrum has changed, with the peaks for above-threshold ionization now less visible for $M_{L}=1$ compared to $M_{L}=0$. In addition, the peak at $4 \mathrm{eV}$ appears to be affected by an interference effect for $M_{L}=0$, whereas no such effect is seen for $M_{L}=1$. The origin of this interference structure is unclear. The inclusion of higher-lying thresholds ensures that $\mathrm{C}$ resonances can be present at this energy, but only states of ${ }^{3} S^{e}$ symmetry would be accessible for $M_{L}=0$ and not for $M_{L}=1$.

The ejected-electron momentum distributions shown in Figs. 1 and 2 can be used to model the influence of spin-orbit dynamics. In experiment [4,5], an electron is detached from $\mathrm{C}^{-}$to leave a residual $\mathrm{C}$ atom. This $\mathrm{C}$ atom is in a superposition of the different $J$ levels of the $2 s^{2} 2 p^{2}$ ground state, and this superposition will evolve in time due to the spin-orbit splitting of the different $J$ levels. The evolution of the $\mathrm{C}$ atom is measured experimentally by examining the dependence of the emission of high-energy electrons along the laser 
polarization axis on time delay. The polarization axis of the $\mathrm{C}^{-}$photodetachment laser is varied between perpendicular and parallel polarization, and the dynamics is determined by examining the difference in $\mathrm{C}^{+}$yield along the probe laser polarization axis between the two polarizations for the pump laser.

In our theoretical model, we assume that the pump laser detaches the $m_{\ell}=0$ electron from the $2 p^{3}{ }^{4} S^{o}$ ground state of a initial $\mathrm{C}^{-}$ion, leaving a $\mathrm{C}$ atom in its ground state with $M_{L}=0$. For parallel polarization of the probe laser, the initial state at zero time delay is thus an $M_{L}=0$ state, whereas for perpendicular polarization, the initial state is in a superposition of $M_{L}=1$ and $M_{L}=-1$. We can then project the ${ }^{3} P^{e}$ initial state with quantum numbers $L, M_{L}, S, M_{S}$ onto the $J, M_{J}$ sublevels. We then propagate these $J, M_{J}$ sublevels in time according to the energy splittings given in the literature [9]. After a time delay, we project back onto the $L, M_{L}, S, M_{S}$ sublevels and use the population in the different $M_{L}$ levels to predict the ejected-electron momentum distribution. The emission within a cone of $11.7^{\circ}$ from the laser polarization axis, with a minimum magnitude for the momentum of 0.4 atomic units, similar to experiment [4,5], is then taken as the high-energy-ionization yield along the laser polarization direction for both the initial state in $M_{L}=0$ or in the superposition for $M_{L}= \pm 1$. The propagation of the $\mathrm{C}$ ground state during the time delay can give rise to three beat periods in the picosecond range, $\tau_{1}=1235 \mathrm{fs}, \tau_{2}=769 \mathrm{fs}$, and $\tau_{3}=2034 \mathrm{fs}$.

Figure 5 shows rescaled normalized high-energyionization-yield differences, similar to the definition used in the experimental study [5],

$$
S(\tau)=\frac{S_{ \pm 1}(\tau)-S_{0}(\tau)}{S_{ \pm 1}(\tau)+S_{0}(\tau)},
$$

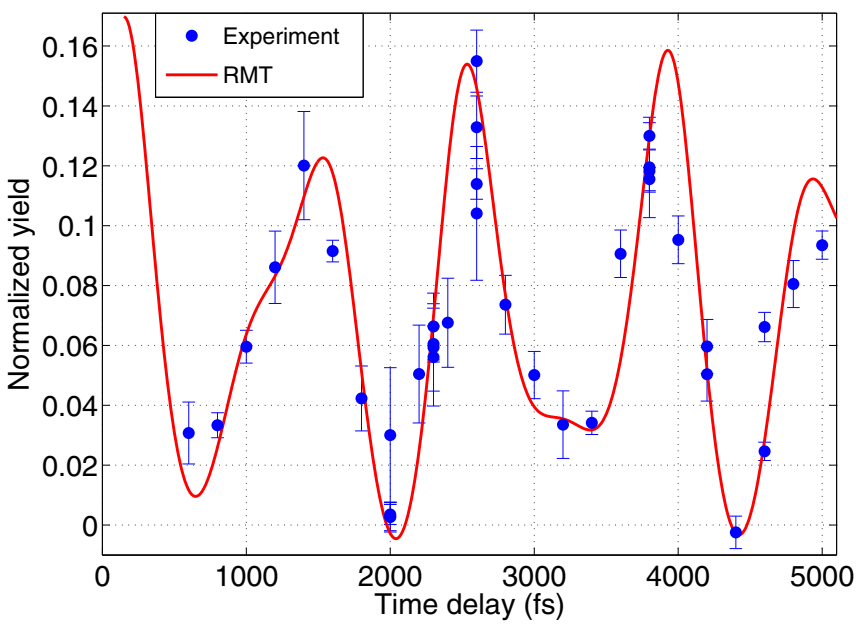

FIG. 5. (Color online) Normalized high-energy-ionization-yield differences as a function of time delay for $\mathrm{C}$. The experimental data (circles [5]) shows normalized ionization yield differences for energetic electrons along the probe-laser polarization axis between a probe pulse with perpendicular polarization direction and parallel polarization direction. The theoretical data shows ionization-yield differences for energetic electrons along the probe-laser polarization axis between $\mathrm{C}$ atoms with $M_{L}=1$ and $M_{L}=0$ at zero time delay. where $S_{ \pm 1}(\tau)$ is the high-energy-ionization yield along the laser polarization axis obtained for the initial superposition of $M_{L}= \pm 1$ at a time delay $\tau$, and $S_{0}(\tau)$ the same obtained for $M_{L}=0$. The high-energy-ionization yields have been rescaled to match up with the experimental results in overall variation of the normalized yields, zero yield position and zero time delay. The first reason for this rescaling is that the experimental study was carried out under significantly different experimental conditions: the wavelength was $1310 \mathrm{~nm}$, and the peak intensity was estimated to be $4 \times 10^{14} \mathrm{~W} / \mathrm{cm}^{2}$. This may lead to significant differences in the actual ionization yields. The second reason is the assumption of pure $m_{\ell}=0$ emission by the pump pulse. Although the emission of $m_{\ell}=0$ electrons should dominate, some $m_{\ell}= \pm 1$ electron will also be emitted. However, these differences will not affect the fundamental character of the dynamics induced by the spin-orbit interaction: the same dynamics should be observed in both theory and experiment.

Figure 5 indeed shows excellent agreement between the experimentally observed effect of spin-orbit dynamics on the ejected-electron momentum distributions and the theoretical model. This demonstrates that it is appropriate to separate the dynamics induced by the laser field from the dynamics induced by the spin-orbit interaction for this particular investigation. The good agreement further demonstrates that the main physical reason that the spin-orbit dynamics can be observed in this scheme, is that the emission of $2 p$ electron with $m_{\ell}=0$ is forbidden when the $\mathrm{C}$ atom is in a state with $M_{L}=0$.

\section{CONCLUSIONS}

We have developed capability within the $R$-matrix-withtime-dependence approach to investigate multiphoton ionization of general atoms with nonzero initial magnetic quantum number. The size of the calculations approximately double due to the need to take both parities into account for each angular momentum. We demonstrate the capability of the approach by investigating multiphoton ionization of ground-state $\mathrm{C}$ atoms at a wavelength of $390 \mathrm{~nm}$. The ejected-electron momentum distributions show that both $2 p$ and $2 s$ electrons can be ejected during the process with the emission of $2 s$ electrons gaining in importance for initial $M_{L}=0$ compared to $M_{L}=1$. For an initial state with $M_{L}=0$, emission of $2 p$ electrons cannot occur along the laser polarization axis, and this provides an excellent means of demonstrating the dynamics induced by spin-orbit coupling in the $\mathrm{C}$ ground state. A theoretical model of this dynamics shows excellent agreement with the experimentally observed dynamics.

In the calculations, we have used significant expansion lengths in the description of $\mathrm{C}$. We have used eight target states and a maximum angular momentum $L_{\max }=53$. The calculations demonstrate that the RMT codes are capable of handling larger basis sets and more extensive angularmomentum expansions, including outer-region expansions of well over 1000 channels. Application of the RMT codes to problems involving significant CI expansions in the inner region is therefore possible. This would be important for the detailed treatment of ultrafast processes in inner shells, where changes to the outer-electron orbitals following inner-shell emission may have to be included. 


\section{ACKNOWLEDGMENTS}

The authors would like to thank M. Eklund and I. Yu. Kiyan for providing their data in numerical form. They would further like to thank J. S. Parker and K. T. Taylor for valuable discussions. The RMT codes were primarily developed by M. A. Lysaght and L. R. Moore. This research has been supported by the Initial Training Network CORINF under a Marie Curie Action of the European Commission and by the UK Engineering and Physical Sciences Research Council under Grant No. G/055416/1. This work made use of the facilities of HECToR, the UK's national high-performance computing service, which is provided by UoE HPCx Ltd. at the University of Edinburgh, Cray Inc., and NAG Ltd, and funded by the Office of Science and Technology through EPSRC's High-End Computing Programme.
[1] F. Krausz and M. Ivanov, Rev. Mod. Phys. 81, 163 (2009).

[2] A. Fleischer, H. J. Wörner, L. Arissian, L. R. Liu, M. Meckel, A. Rippert, R. Dörner, D. M. Villeneuve, P. B. Corkum, and A. Staudte, Phys. Rev. Lett. 107, 113003 (2011).

[3] E. Goulielmakis et al., Nature (London) 466, 739 (2010).

[4] H. Hultgren, M. Eklund, D. Hanstorp, and I. Yu. Kiyan, Phys. Rev. A 87, 031404(R) (2013).

[5] M. Eklund, H. Hultgren, D. Hanstorp, and I. Yu. Kiyan, Phys. Rev. A 88, 023423(R) (2013).

[6] E. Sokell, S. Zamith, M. A. Bouchene, and B. Girard, J. Phys. B: At., Mol. Opt. Phys. 33, 2005 (2000).

[7] A. C. Brown and H. W. van der Hart, Phys. Rev. A 88, 033419 (2013).

[8] O. Hassouneh, A. C. Brown, and H. W. van der Hart, Phys. Rev. A 89, 033409 (2014).

[9] C. E. Moore, in CRC Series in Evaluated Data in Atomic Physics, edited by J. W. Gallagher (CRC Press, Boca Raton, 1993).

[10] N. Rohringer and R. Santra, Phys. Rev. A 79, 053402 (2009).

[11] S. Pabst, A. Sytcheva, A. Moulet, A. Wirth, E. Goulielmakis, and R. Santra, Phys. Rev. A 86, 063411 (2012).

[12] L. A. A. Nikolopoulos, J. S. Parker, and K. T. Taylor, Phys. Rev. A 78, 063420 (2008).

[13] M. A. Lysaght, H. W. van der Hart, and P. G. Burke, Phys. Rev. A 79, 053411 (2009).
[14] M. A. Lysaght, L. R. Moore, L. A. A. Nikolopoulos, J. S. Parker, H. W. van der Hart, and K. T. Taylor, in Quantum Dynamic Imaging: Theoretical and Numerical Methods, edited by A. D. Bandrauk and M. Ivanov (Springer, Berlin, 2011), pp. 107-134.

[15] L. R. Moore, M. A. Lysaght, L. A. A. Nikolopoulos, J. S. Parker, H. W. van der Hart, and K. T. Taylor, J. Mod. Opt. 58, 1132 (2011)

[16] S. Hutchinson, M. A. Lysaght, and H. W. van der Hart, J. Phys. B: At., Mol. Opt. Phys. 44, 215602 (2011).

[17] L. R. Moore, M. A. Lysaght, J. S. Parker, H. W. van der Hart, and K. T. Taylor, Phys. Rev. A 84, 061404 (2011).

[18] H. W. van der Hart, Phys. Rev. A 89, 053407 (2014).

[19] P. G. Burke and K. A. Berrington, Atomic and Molecular Processes: An R-Matrix Approach (IOP Publishing, Bristol, 1993).

[20] P. G. Burke, R-Matrix Theory of Atomic Collisions (Springer, Heidelberg, 2011).

[21] E. S. Smyth, J. S. Parker, and K. T. Taylor, Comput. Phys. Commun. 114, 1 (1998).

[22] P. G. Burke and K. T. Taylor, J. Phys. B: At. Mol. Phys. 12, 2971 (1979).

[23] E. Clementi and C. Roetti, At. Data Nucl. Data Tables 14, 177 (1974). 